\title{
A Nicotiana benthamiana AP2/ERF transcription factor confers resistance to Phytophthora parasitica
}

\author{
Jing $\mathrm{Yu}^{1,2}$, Chunyue Chai ${ }^{1,3}$, Gan Ai ${ }^{1}$, Yuling Jia ${ }^{1}$, Wenjing Liu ${ }^{3}$, Xiong Zhang ${ }^{1}$, Tian Bai ${ }^{1}$ and Daolong Dou ${ }^{1 *}$ (D)
}

\begin{abstract}
Diseases caused by Phytophthora species seriously affect global crop production and food security. Identification of key factors involved in plant resistance is valuable for disease management. Previously, we characterized the transcriptome of Nicotiana benthamiana which was infected with Phytophthora parasitica. Here, we selected NbERF173, one of the most strongly up-regulated genes of $N$. benthamiana in response to $P$. parasitica infection, for further investigation. First, NbERF173 encodes a conserved transcription factor in our tested plant species. Second, overexpression of NbERF173 in N. benthamiana enhanced its resistance to P. parasitica, and silencing of NbERF173 significantly promoted the infection of both P. parasitica and Botrytis cinerea. Additionally, we demonstrated that NbERF173 can participate in reprograming of gene expression during P. parasitica infection and manipulate expression patterns of many defense-related genes, including two proteinase inhibitors encoding genes (P/1-B and KT/1). Furthermore, overexpression of P/1-B and KT/1 strengthened plant resistance to $P$. parasitica and partially restored the deficiency in resistance of NbERF173-silenced $N$. benthamiana plants. Finally, we found that NbERF173 could not bind to the promoters of P/1-B and KT/1 using yeast one-hybrid assay. Together, our results suggest that NbERF173 positively regulate the disease resistance, probably by reprograming of defense-related genes.
\end{abstract}

Keywords: Nicotiana benthamiana, Phytophthora parasitica, AP2/ERF, Proteinase inhibitor

\section{Background}

Phytophthora species belong to oomycetes and cause devastating diseases in numerous important agricultural and ornamental crops (Kroon et al. 2012; Kamoun et al. 2015). Increasing attention has been focused on Phytophthora parasitica (syn. Phytophthora nicotianae Breda de Haan), which has a broad host range and is responsible for serious damage to agricultural production and natural ecosystems (Meng et al. 2014). For disease management, the most effective method is to breed disease-resistant cultivars. However, the available resistance resource for $P$. parasitica is still limited and the resistance-related genes are rarely reported.

Nicotiana benthamiana, a highly tractable experimental model organism for research of plant pathology, appears to adopt a distinct strategy that is avoidance rather

\footnotetext{
* Correspondence: ddou@njau.edu.cn

${ }^{1}$ Department of Plant Pathology, Nanjing Agricultural University, Nanjing 210095, China

Full list of author information is available at the end of the article
}

than resistance in response to pathogens. A mutation that occurred one million years ago gave rise to a dominant resistance-related gene (Rdr1) in $N$. benthamiana, enabling the plant to grow faster by shortening its growth cycle, although it possesses low resistance to most pathogenic organisms (Bally et al. 2015). $N$. benthamiana is also a frequently-used model plant for studying the interactions between hosts and Phytophthora species because it is highly sensitive to Phytophthora pathogens, and it is easy to implement virusinduced gene silencing and transient expression assays in this plant (Goodin et al. 2008). This phytopathology system will help us to understand not only the pathogenesis of Phytophthora pathogens but also the resistance mechanism of plants.

AP2/ERF (APETALA2/ethylene-responsive element binding factor) transcription factors belong to one of the largest plant transcription factor families, which are characterized by the conserved AP2/ERF DNA binding domain of 57-66 amino acid residues in length 
(Okamuro et al. 1997). Originally, the AP2/ERF family was reported to only exist in the plant kingdom, but recent studies have indicated that AP2/ERF genes are also present in protists and ciliates (Rashid et al. 2012; Licausi et al. 2013). The AP2/ERF family consists of four subfamilies (AP2, CBF/DREB, ERF, and RAV) based on their sequence similarities and numbers of AP2/ERF domain (Sakuma et al. 2002). Most proteins in the ERF family have a single AP2 domain (Nakano et al. 2006). Nevertheless, the AP2 family consists of members characterized by tandem repeats of one or two AP2 domains (Shigyo and Ito 2004). The RAV family proteins possess an ERF domain associated with a B3 DNA-binding domain (Swaminathan et al. 2008). A wide variety of ERF genes have been characterized, including Arabidopsis thaliana AtERF1-5 (Fujimoto et al. 2000), Lycopersicum esculentum LeERF1-4 (Tournier et al. 2003), Glycine max GmERF3 (Zhang et al. 2008) and Petunia hybrid PhERF2/3 (Liu et al. 2011). But their functions in $N$. benthamiana disease resistance are largely unknown.

Plant defense proteins, including proteinase inhibitors, play an important role in plant defense response, which have been well documented (Haq et al. 2004; Kim et al. 2009; Valdes-Rodriguez et al. 2010). For example, Proteinase inhibitors possess potent broad-spectrum growth-inhibitory activity against various plant pathogens (Ryan 1990; Kim et al. 2009). Transgenic rice plants expressing a potato proteinase inhibitor gene displayed enhanced resistance to two pathogens, Magnaporthe oryzae and Fusarium verticillioides by inhibiting fungal carboxypeptidases (Quilis et al. 2007). A pair of genes is highly induced by pathogen infection in both tobacco and tomato, and the corresponding proteins cause lysis of sporangia and growth inhibition of $P$. infestans (Woloshuk et al. 1991). P. infestans also cause an accumulation of serine protease inhibitors in potato tubers (Valueva et al. 1998; Valueva et al. 2003), but their roles in pathogen inhibition are not determined yet. Stacking two protease-inhibitor genes in transgenic tobacco plants showed resistance to both insects and pathogens, including Pythium aphanidermatum infection (Senthilkumar et al. 2010).

Previously, we found that many $N$. benthamiana ERF transcription factors were up-regulated during $P$. parasitica infection using RNA-Seq analysis (Shen et al. 2016), suggesting their potential role in resistance to Phytophthora pathogens. Here, we selected an ERF family member (NbERF173) that was strongly induced by $P$. parasitica infection for further characterization. Results revealed that overexpression or down-regulation of NbERF173 altered the resistance levels to $P$. parasitica in transgenic $N$. benthamiana plants. Furthermore, we demonstrated that down-regulation of NbERF173 could lead to constitutive expression impairment of two proteinase inhibitor encoding genes. Overall, these results indicate that $N b E R F 173$ positively regulate disease resistance in $N$. benthamiana by affecting expression levels of defense-related genes.

\section{Results}

Characterization of NbERF173 in N. benthamiana during $P$. parasitica infection

To precisely analyze the functions of ERFs, we designated all 266 ERF transcription factors in $N$. benthamiana as NbERF1 to NbERF266 (Additional file 1: Table S1). Based on the RNA-Seq data (Shen et al. 2016), we selected 64 genes that were up-regulated with $>2$-folds changes upon $P$. parasitica infection for further analysis (Additional file 2: Table S2). Total RNA was isolated from $N$. benthamiana seedlings infected with $P$. parasitica zoospores at $0,3,6$, and $9 \mathrm{~h}$ post inoculation (hpi), and used for further confirmation of the upregulated $E R F$ genes through RT-PCR with gene-specific primers. We successively validated their expression patterns by using qRT-PCR, and finally $9 E R F$ genes were successfully confirmed as up-regulated genes, including NbERF173 (Additional file 2: Table S2). Furthermore, qRT-PCR results showed that expression of NbERF173 was also significantly induced in response to $P$. parasitica infection and reached a peak at 6 hpi (Fig. 1a). Since NbERF173 was up-regulated by more than 250 folds (Fig. 1a), we selected NbERF173 for further functional characterization.

To isolate the full-length cDNA of NbERF173, we searched the corresponding protein in $N$. benthamiana genome using the gene ID (Niben101Scf01430g00007.1), and then confirmed by RT-PCR. Analysis of the obtained sequence (https://web.expasy.org/compute_pi/) revealed that NbERF173 contained a 780-bp open reading frame encoding a 259 -aa protein with a predicted molecular mass of $29.27 \mathrm{kDa}(\mathrm{pI}=5.64)$. NbERF173 contains multiply nuclear localization signals (NLSs) predicted by software (http://nls-mapper.iab.keio.ac.jp/) and a nuclear export signal (NES) at residues from 203 to 212 using NetNES1.1 Server (http://www.cbs.dtu.dk/services/NetNES-1.1). Sequence blast and alignment showed that NbERF175, NbERF236, NbERF244, and NbERF246 are homologs of NbERF173 in N. benthamiana, but none of them exhibited induced expression during infection. Furthermore, NbERF173 shared varying degrees of similarity with other plant ERFs. We constructed a phylogenetic tree of these ERF proteins using the alignment results with the MEGA7 NJ method (Tamura et al. 2011). As shown in Fig. 1b, NbERF173 shared similarity of $86 \%$ with NtERF071 from N. tabacum, similarity of $73 \%$ with CaERF071 (Lee et al. 2004), 59\% with SIERF2 (Tournier et al. 2003; Pirrello et al. 2006), and 50\% with AtERF (NP_188299.1) from Arabidopsis (Buttner and Singh 1997) (Fig. 1b). As shown in Fig. 1c, NbERF173 was predicted to contain a conserved 


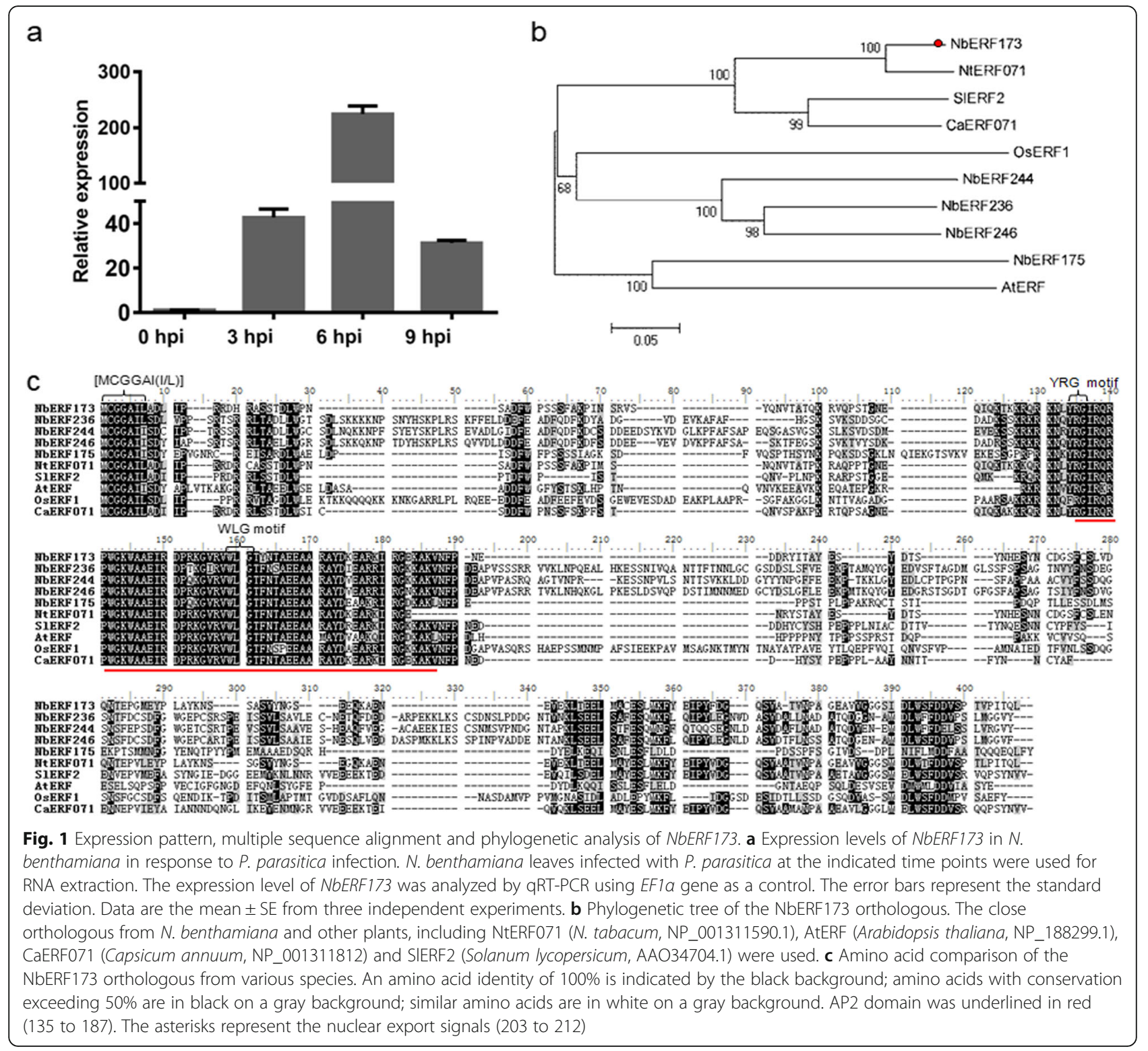

AP2/ERF DNA binding domain with 52-aa residues, suggesting that NbERF173 belongs to the AP2/ERF family (Hao et al. 1998). It also contains a conserved [MCGGAI(I/L)] motif in the N-terminal region, suggesting that it belongs to the class IV of ERF family (Tournier et al. 2003). Interestingly, it has a conserved WLG motif, which exists only in a few ERFs (Li et al. 2015). The expression of this gene was induced by hormones, such as salicylic acid, jasmonic acid and ethylene (Additional file 3: Figure S1).

Overexpression of NbERF173 in N. benthamiana enhanced its disease resistance

To investigate the function of NbERF173 in plant disease resistance, we fused its full-length cDNA with a GFP-tag sequence at the $\mathrm{N}$-terminus and generated a construct, pBINPLUS:GFP:NbERF173. Agrobacterium tumefaciensmediated transient expression assay was implemented to express the GFP:NbERF173 fusion protein in $N$. benthamiana leaves. GFP protein alone, as a negative control, localized in both cytoplasm and nucleus, while the GFP:NbERF173 fusion protein localized only in the nucleus, which was confirmed by DAPI staining (Fig. 2a). After transient expression, the corresponding $N$. benthamiana leaves were inoculated with zoospore suspensions of $P$. parasitica. The lesion diameters were measured at 36 and 48 hpi. In contrast to the GFP expression control on one side of the leaves, expression of NbERF173 on the other side developed significantly smaller lesions (Fig. 2b, c). These results indicated that 


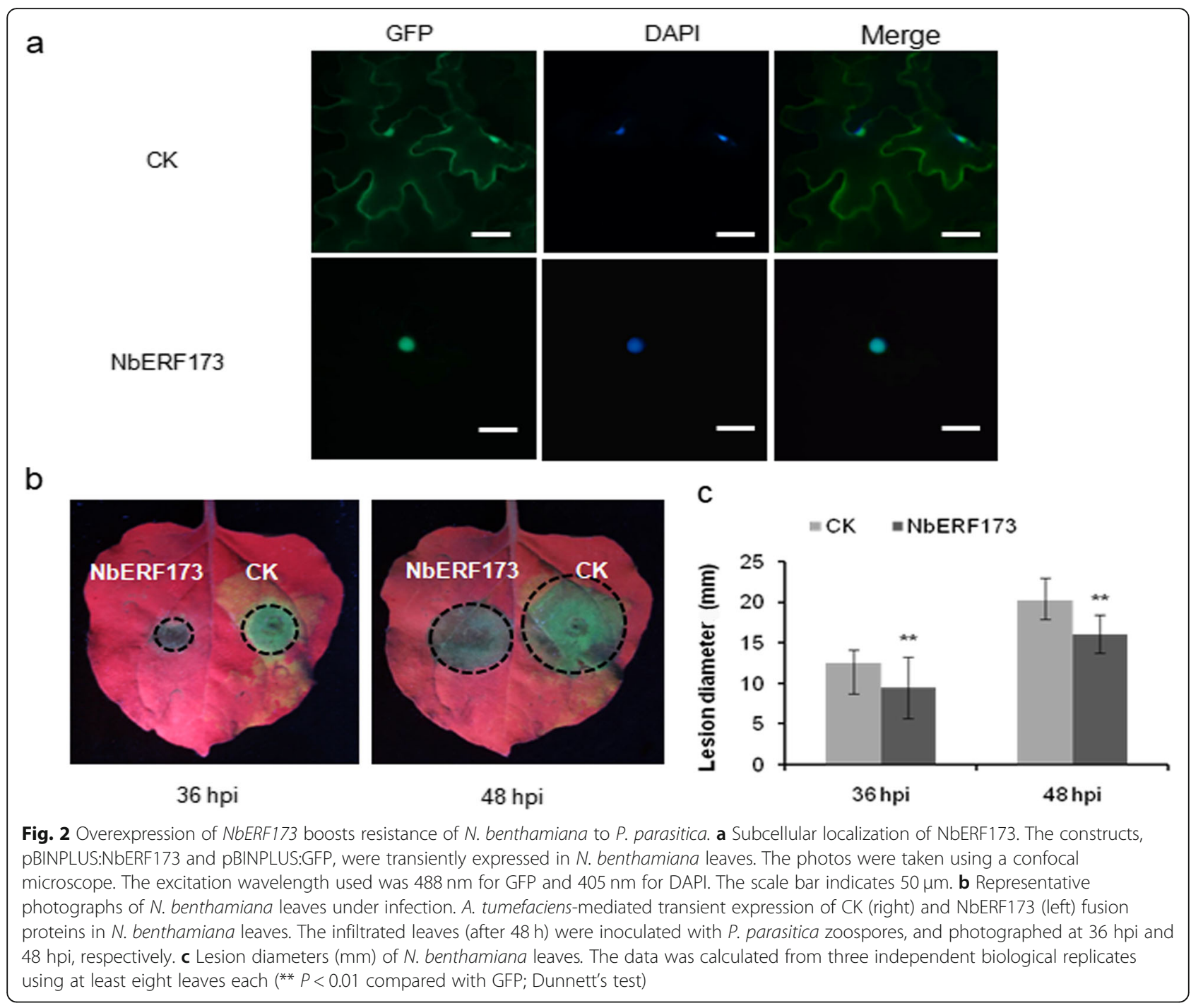

overexpression of NbERF173 enhanced disease resistance to $P$. parasitica in $N$. benthamiana.

\section{Silencing of NbERF173 impaired the disease resistance of $N$. benthamiana}

Next, we silenced NbERF173 by using virus-induced gene silencing (VIGS) assay in N. benthamiana. qRTPCR analysis showed that the expression level of NbERF173 was reduced by more than $96 \%$ in the TRV: NbERF173 plants (Fig. 3a). In contrast, other homologous genes including NbERF175, NbERF236, NbERF244 and NbERF246 were not affected in the NbERF173-silenced plants, indicating the high specificity of gene silencing of NbERF173 (Additional file 4: Figure S2). It is worth mentioning that NbERF173-silenced $N$. benthamiana seedlings displayed normal growth phenotype (Additional file 4: Figure S2). Afterwards, NbERF173-silenced $N$. benthamiana leaves were inoculated with $P$. parasitica zoospore suspensions. As shown in Fig. 3b, lesion diameters of the silenced leaves (TRV:NbERF173) were significantly larger than those of the controls (TRV:GFP) at 36 and 48 hpi (Fig. 3b). To dissect pathogen development, we stained the inoculated tissue by trypan blue to visualize hyphal extension in host cells. Consist with the above results, abundant $P$. parasitica hyphae were observed in TRV:NbERF173 leaves while few hyphae were present in the control leaves (TRV:GFP) (Fig. 3c). Taken together, these results suggested that $N b E R F 173$ is required for the resistance of $N$. benthamiana to $P$. parasitica. To further analyze the resistance conferred by NbERF173, we inoculated NbERF173-silenced $N$. benthamiana leaves with Botrytis cinerea, a typical necrotrophic fungal pathogen, and found that NbERF173-silenced plants also showed more sensitivity than the control leaves to $B$. cinerea infection (Fig. 3d). Collectively, NbERF173 positively regulates the resistance of $N$. benthamiana to plant pathogens. 


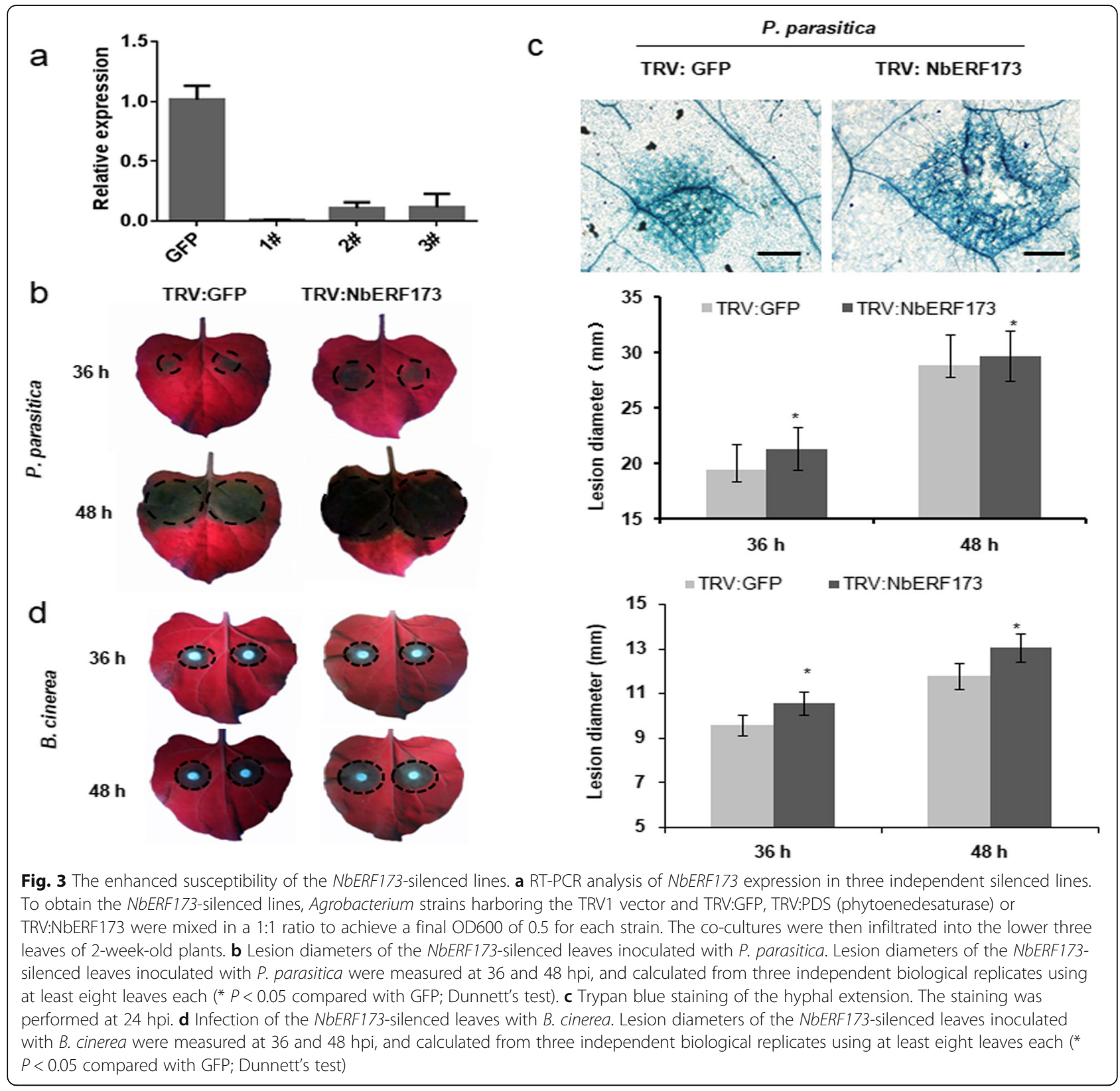

\section{Analysis of genes regulated by NbERF173 during $P$. parasitica infection}

To investigate the mechanism underlying the plant defense conferred by NbERF173, we compared the gene expression profiling between NbERF173-silenced and non-silenced plants at 0 and 6 hpi upon $P$. parasitica infection by using Illumina-based RNA-Seq assay. In the T6 treatment ( $P$. parasitica infected $N$. benthamiana wild-type leaves for $6 \mathrm{~h}$ ), 6205 genes (5364, 770, 60 and 11) were up-regulated and 8154 (6736, 895, 486 and 37) genes were down-regulated compared with T0 (the noninfected control leaves) (2-fold changes, $P<0.05$ ) (Fig. 4a). Among the up-regulated $N$. benthamiana genes upon infection, we found that expression of only 71 genes (60 and 11) were repressed in the E0-T0 (NbERF173-silenced lines compared with the wild type without pathogen infection), and 781 genes (770 and 11) were down-regulated in the E6-T6 (NbERF173-silenced lines compared with the wild type upon pathogen infection for $6 \mathrm{~h}$ ). Finally, we obtained 11 candidate genes (Additional file 5: Table S3) that are induced by pathogen, but suppressed in the NbERF173-silenced lines in both 0 and 6 hpi (Fig. 4a), including two proteinase inhibitors and two heat shock proteins. These genes were designated as defense-related genes that are positively regulated by NbERF173. Among the $8154 \mathrm{~N}$. 


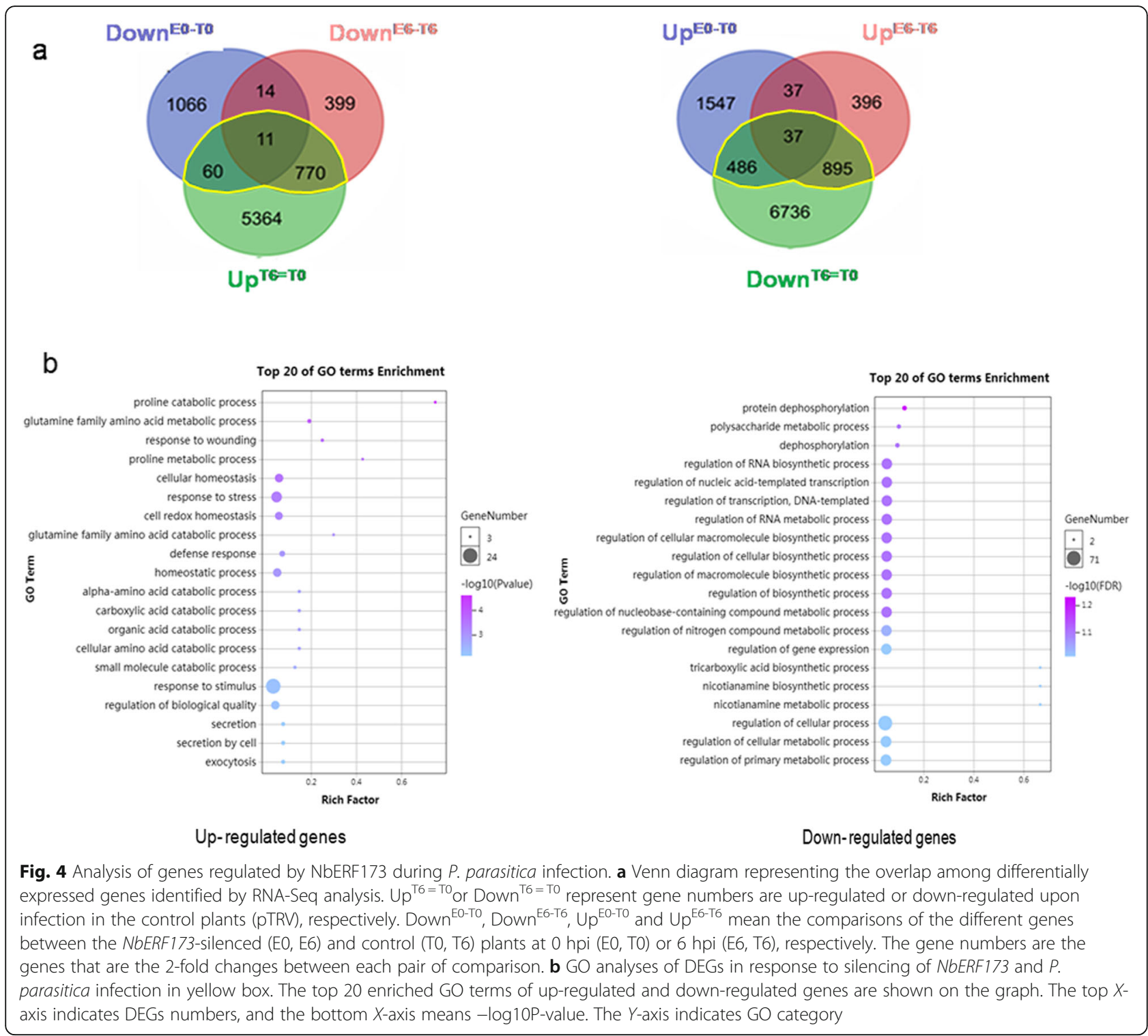

benthamiana genes down-regulated by pathogen, 523 genes (486 and 37) were up-regulated in the E0-T0 (NbERF173-silenced lines compared with the wild type without infection) and 932 genes (895 and 37) were upregulated in the E6-T6 (NbERF173-silenced lines compared with the wild type upon infection). In summary, 37 candidate genes (Additional file 6: Table S4) were negatively expressed upon infection, which could be reversed in the NbERF173-silenced plants. These genes encoded 2-succinylbenzoate-CoA ligase, MYC2 and NAC36 transcription factors. Collectively, a large number of genes are involved in the infection process, and we suggest that NbERF173 plays an important role in regulation of gene expression.

Next, GO enrichment analysis was performed to identify differentially expressed gene sets in response to silencing of NbERF173 and P. parasitica infection based on GO category. The up-regulated genes on the left pattern of Fig. $4 \mathrm{~b}$ showed genes that were both up-regulated in $P$. parasitica infected $N$. benthamiana and down-regulated in NbERF173-silenced lines (770, 60 and 11). The down-regulated genes on the right pattern of Fig. 4b showed genes that were both down-regulated in $P$. parasitica infected $N$. benthamiana and up-regulated in NbERF173-silenced lines (895, 486 and 37). The top 20 enriched GO terms were determined (Fig. 4b), among which "response to stress and stimulus" was the most abundant term in the up-regulated GO categories, while "regulation of biosynthetic process" and "regulation of cellular process" were the dominant terms in the down-regulated genes. 
Expression of two proteinase inhibitor genes was repressed in NbERF173-silenced lines

Based on RNA-Seq results, we selected 7 up-regulated and 5 down-regulated genes involved in defense responses for qRT-PCR validation (Additional file 7: Table S5). The results manifested that these genes exhibited similar expression patterns compared with those derived from RNA-Seq approach (Fig. 5), highlighting the accuracy and reproducibility of the RNA-Seq analysis in this study. As shown in Fig. 5, expression levels of 4 genes were significantly $(P<0.01)$ reduced in the NbERF173-silenced lines compared with the non-silenced lines at 0 and $6 \mathrm{hpi}$, which encode proteinase inhibitor I-B (PI1-B), myb-related protein
305 (MYB305), Kunitz trypsin inhibitor 1 (KTI1) and chaperone protein HtpG, respectively. Notably, the expression levels of two protease inhibitor genes in NbERF173-silenced $N$. benthamiana were less than one-fifth of those in control plants (pTRV:GFP). In the previous RNA-Seq data (Shen et al. 2016), PI1-B and KTI1 were also up-regulated upon $P$. parasitica infection, suggesting their potential roles in plant defense response. Furthermore, we demonstrated that the expression of PI1-B and KTI1 in NbERF173-overexpressed leaves was up-regulated by performing qRTPCR assay (Additional file 8: Figure S3). Taken together, these results suggested that NbERF173 tightly regulates the expression of PI1-B and KTI1.

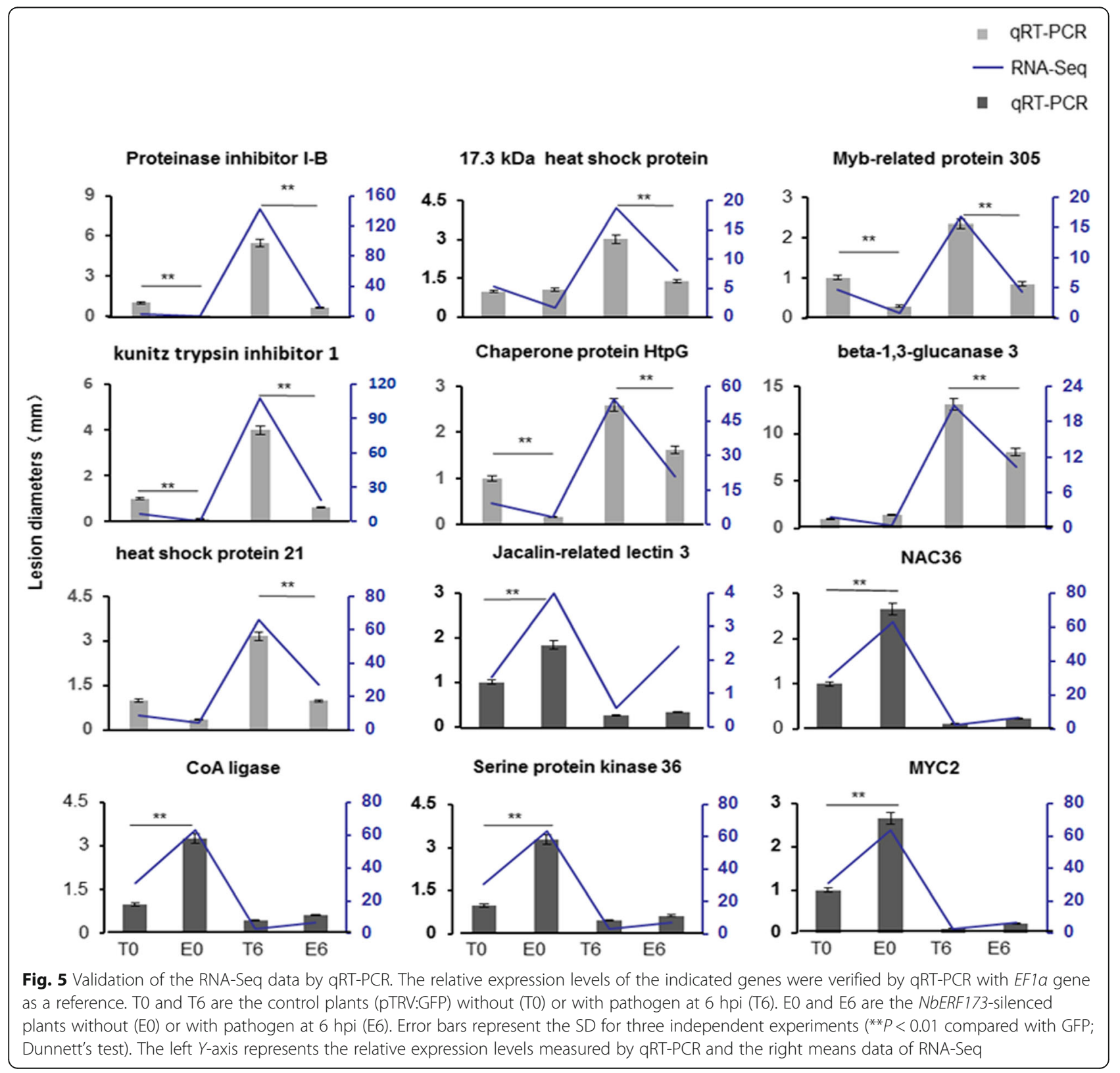


Since proteinase inhibitors were reported to play an important role in defense response against pathogens (Carrillo et al. 2011; Majeed et al. 2011), we speculated that NbERF173 possibly confers the resistance to $P$. parasitica through positively regulating the expression of PI1-B and KTI1 in N. benthamiana. To verify our hypothesis, we firstly performed $A$. tumefaciens-mediated transient expression of these two genes in N. benthamiana. Afterwards, we found that overexpression of these two PIs (PI1-B and KTI1) in N. benthamiana enhanced plant resistance to $P$. parasitica (Fig. 6). Moreover, the deficiency of NbERF173 gene expression significantly reduced the resistance of $N$. benthamiana to $P$. parasitica (Fig. 3b), but overexpression of thses two PIs in NbERF173-silenced lines could partially restore the resistance to $P$. parasitica (Fig. 6). Therefore, these results indicated that NbERF173 confers resistance to $P$. parasitica probably by regulating two PI genes expression.

\section{NbERF173 has no binding activities to the promoters of two proteinase inhibitors}

To determine whether there are direct physical interactions between NbERF173 and the promoters of two PI genes (Additional file 9: Table S6), we conducted the yeast one-hybridization $(\mathrm{Y} 1 \mathrm{H})$ experiment. In the $\mathrm{Y} 1 \mathrm{H}$ assay, yeast transformant harboring pGADT53m $\times$ pHIS53 serves as a positive control, and pGADT53m $\times$
pHIS2 serves as a negative control. The recombinant bait plasmids by pGAD-NbERF173 $\times$ pHIS2, pGAD empty $\times$ pHIS2-PPI1-B (promoter region of the PI1-B gene) and pGAD empty $\times$ pHIS2-KTI-B (promoter region of the KTI gene) did not grow well in medium without histidine, suggesting they didn't have selfactivating activities. Unfortunately, yeast transformants harboring pGADT7-NbERF173 $\times$ pHIS2-PPI1-B or pGADT7-NbERF173 $\times$ pHIS2-KTI-B also did not grow well in medium without histidine (Additional file 10: Figure S4), indicating that NbERF173 could not bind the promoters of the two PI genes. In other words, NbERF173 may indirectly regulate the two PI genes expression or need other components as partners to accomplish its function.

\section{Discussion}

Previous studies on ERF genes are mainly focused on their involvement in plant growth and development. For example, overexpression of rice OsERF1 affects growth and development in Arabidopsis ( $\mathrm{Hu}$ et al. 2008), SIERF2, a tomato ethylene response factor, is involved in seed germination (Pirrello et al. 2006). ERFs also function in transcriptional regulation of ethylene biosynthesis and hormone responses. For example, SIERF2 is involved in ethylene response (Pirrello et al. 2006). In addition, TERF2/LeERF2 enhances tomato tolerance to freezing

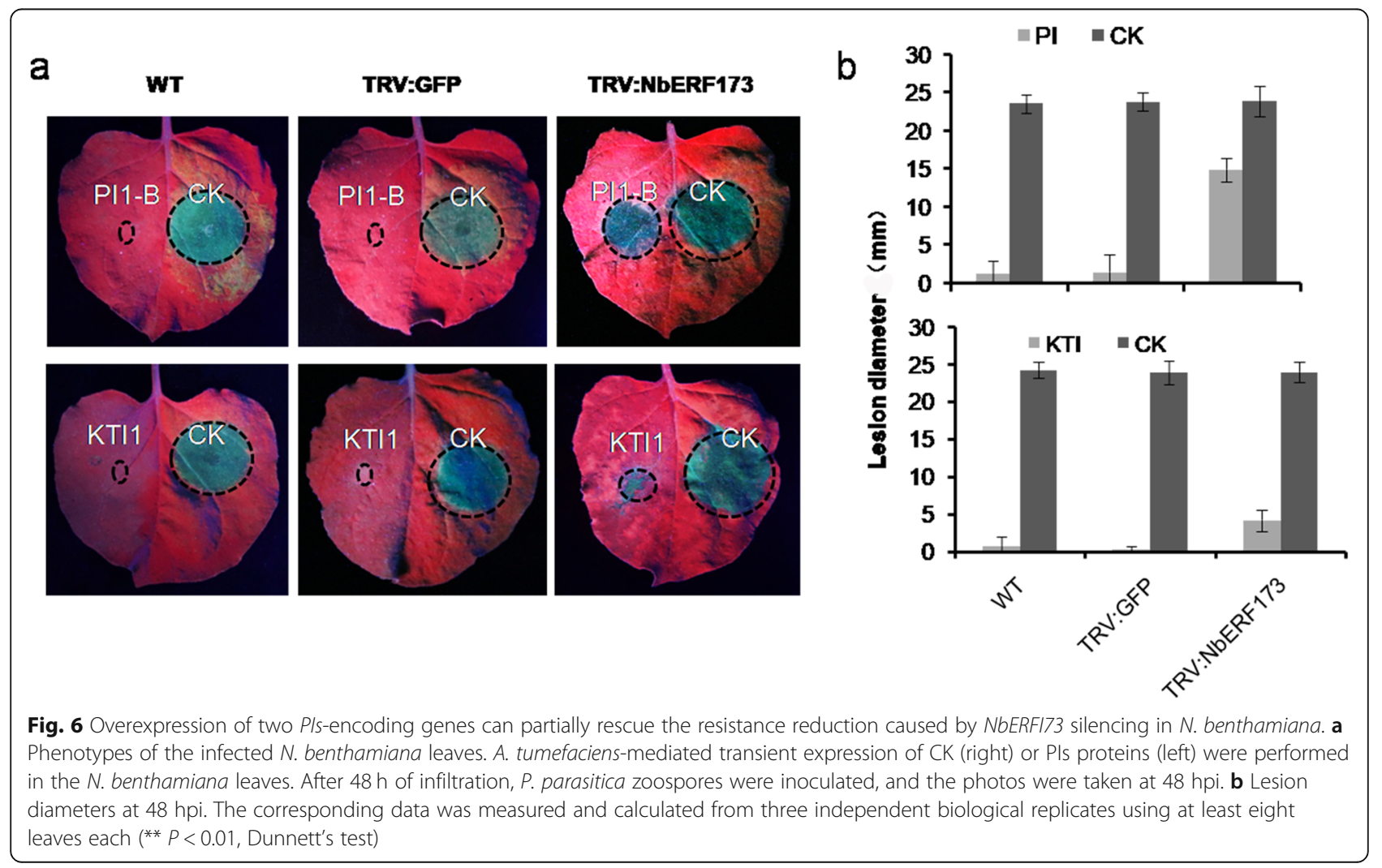


(Zhang and Huang 2010). However, ERFs that function in disease resistance are not fully studied, especially in $N$. benthamiana. In this study, we systematically identified the $N$. benthamiana ERFs, and found NbERF173 conferred $N$. benthamiana resistance to $P$. parasitica with induced expression in the early stages during infection. Overexpression of NbERF173 in N. benthamiana enhanced its resistance to $P$. parasitica. Furthermore, suppressed expression of NbERF173 by VIGS led to a dramatic reduction in $N$. benthamiana resistance to $P$. parasitica and $B$. cinerea. These results suggested that NbERF173 participates in $N$. benthamiana resistance to pathogens.

Some ERFs are involved in plant defense responses against pathogen infection by regulating different gene sets, including pathogenesis-related genes (Shin et al. 2002; Gutterson and Reuber 2004). For example, CaPTI1 from Capsicum annuum is involved in the regulation of defense response to $P$. capsici (Jin et al. 2015). The wheat pathogen-induced ERF transcription factor TaPIE1 positively mediates host responses to a necrotrophic pathogen Rhizoctonia cerealis (Zhu et al. 2014). Interestingly, it also regulates resistance of freezing stress by activating a subset of defense- and stress-related genes downstream of the ethylene signaling pathway. Our results revealed that NbERF173 acted as a positive regulator for defense against both semibiotrophic and necrotrophic pathogens. GmERF5 is a soybean EAR motif-containing ERF and binds to the GCC-box element to act as a repressor of gene transcription. Its overexpression in soybean confers high resistance to $P$. sojae (Dong et al. 2015). Unfortunately, we failed to obtain the target cis-elements of NbERF173 using RNA-seq data, which is perhaps caused by the fact that many genes are indirectly regulated. We found that a majority of genes was negatively regulated by NbERF173, which are enriched in GO terms of "regulation of biosynthetic process" and "regulation of cellular process". It will be interesting to illustrate whether NbERF173 acts as a repressor or activator of gene transcription. Another soybean ERF, GmERF113, also positively regulates $P$. sojae resistance. It interacts with a basic helix-loop-helix transcription factor to control expression of the pathogenesisrelated genes (Zhao et al. 2017). Many defense-related genes were identified to be manipulated by NbERF173 in this study. Additional studies of mechanisms are needed to clarify its direct integration with the cis-elements or combination with other essential regulators.

It has been well documented that PIs possess potent and broad-spectrum growth-inhibitory activity against plant pathogens or insects (Ryan 1990; Haq et al. 2004; Kim et al. 2009; Valdes-Rodriguez et al. 2010). Transgenic plants have been generated and their resistance to pathogens has also been tested (Schlüter et al. 2010).
Numerous reports have underlined the potential of plant PIs for the utilization in genetically modified plants for disease-resistance (Benchabane et al. 2010; Schlüter et al. 2010; Senthilkumar et al. 2010). However, whether these genes are regulated by other components is largely unknown. In our study, NbERF173 affected the expression of defense-related genes. To analyze the mechanism of NbERF173 in conferring the resistance of $N$. benthamiana to $P$. parasitica, we used Illumina-based RNA-Seq assay to analyze gene expression profiling between NbERF173-silenced and non-silenced $N$. benthamiana leaves. Finally, we identified two PI genes in $N$. benthamiana which were positively regulated by NbERF173. We found overexpression of two PIs (PI and $K T I$ ) in wild-type $N$. benthamiana enhanced its resistance to Phytophthora infection, which is consistent with previous studies. Furthermore, overexpression of $P I$ and $K T I$ in NbERF173-silenced leaves partially restored the resistance, suggesting they might act at the downstream of NbERF173. Unfortunately, we found that NbERF173 failed to directly bind to the promoters of $P I$ and KTI. We speculate that NbERF173 may indirectly regulate the two PI genes expression or the regulation needs other component as partners, which need to be addressed in the future.

\section{Conclusions}

In this study, we identified a $N$. benthamiana ERF gene NbERF173 that plays a positive role in plant resistance to Phytophthora pathogens. NbERF173 can regulate expression of many defense-related genes, including proteinase inhibitors. Our results revealed a potential mechanism for NbERF173 regulating the disease resistance of $N$. benthamiana.

\section{Methods}

Microbial strains, plants, and culture conditions

P. parasitica (isolate Pp025) was cultured for 3 days on $2.5 \%$ vegetable (V8) juice medium at $25^{\circ} \mathrm{C}$ in the dark (Dou et al. 2008). To prepare P. parasitica zoospores, the mycelia were cultured in $2.5 \%$ liquid V8 juice medium for 3 days and washed five times with sterilized water at room temperature and incubated at $25^{\circ} \mathrm{C}$ until sporangia formed. To initiate zoospore release, fresh cold sterilized water $\left(4^{\circ} \mathrm{C}\right)$ was added and the plates were incubated at $4{ }^{\circ} \mathrm{C}$. The zoospore concentration was estimated with a hemocytometer. $N$. benthamiana was grown at $25{ }^{\circ} \mathrm{C}$ under a photoperiod of $16 \mathrm{~h}$ light $/ 8 \mathrm{~h}$ dark in an environmentally controlled growth room.

\section{Bioinformatics analysis and primer design}

The produced clean reads were mapped to the $N$. benthamiana V1.0.1 reference genome (https://solgenomics.net/organism/Nicotiana_benthamiana/genome) 
using TopHat (Trapnell et al. 2009) software with default parameters. Only the reads that could be uniquely mapped to the $N$. benthamiana genome were used for subsequent processing. The retained reads were quantified using Cufflinksv1.0.3 program (Trapnell et al. 2009), and the expression level of each gene was calculated by normalizing to the fragment perkilobase of exon per million mapped reads (FPKM) value. Weakly expressed genes were removed, and only genes with FPKM larger than 1 were included in the analysis. Differentially expressed genes were identified using the GFOLD algorithm (Feng et al. 2012), which was biologically meaningful for single replicate experiments. Genes with four fold change (GFOLD [1or-1]) were considered differentially expressed between two samples. MapMan ontology tool was used to obtain an overview of $N$. benthamiana genes involved in metabolic pathway in which a plant-specific ontology classifies genes into hierarchical categories and was denominated BIN (Thimm et al. 2004). N. benthamiana genes were assigned to BINs using the Mercator automated annotation pipeline. Then riched Mapman BINs were identified using Fisher's exact test.

The multiple sequence alignment was constructed by CLUSTALW, and the phylogenetic tree was constructed using the MEGA 5.0 software. Protein domain and motif analyses were conducted using the NCBI conserved domain database and Motif Scan (http://myhits.isb-sib.ch/ cgi-bin/motif_scan). All primers used for RT-PCR, qRTPCR, and plasmid construction were designed by Primer Premier 5 software (Additional file 11: Table S7). The primer specificity was evaluated by sequence similarity comparison. The reaction conditions were optimized by determining the optimal annealing temperature and primer concentration.

The whole genome and protein sequences of $N$. benthamiana were downloaded from the National Center for Biotechnology Information (NCBI). The protein sequences of putative NbERFs in $N$. benthamiana were aligned with the Clustal X program (version 1.83) using the default parameters. Phylogenetic trees were constructed using the aligned result with the NeighborJoining (NJ) method in MEGA7 software (version 7.0.2.6, Tamura et al. 2011). Subcellular localization signal analysis was conducted using NetNES 1.1 Server. (http://www.cbs.dtu.dk/services/NetNES-1.1).

\section{RNA extraction and RT-PCR analysis}

Six-week-old $N$. benthamiana leaves were detached and immersed in suspensions of $P$. parasitica containing $1 \times$ $10^{5}$ zoospores $/ \mathrm{mL}$. Leaves were collected at $0,3,6$, and 9 hpi and frozen in liquid nitrogen. The RNA was extracted with Trizol reagent (TIANGEN), following the manufacturer's instructions, and then treated with DNase I at $37^{\circ} \mathrm{C}$ for $30 \mathrm{~min}$. RNA $(1 \mu \mathrm{g})$ was then reverse-transcribed using a PrimeScript First-strand cDNA Synthesis Kit (TaKaRa) according to the manufacturer's instructions. A $20 \mu \mathrm{L}$ aliquot of cDNA was diluted to $60 \mu \mathrm{L}$ with water, and then $2 \mu \mathrm{L}$ of the diluted cDNA was used for the analyses. For real-time quantitative RT-PCR (qRT-PCR) analysis, gene-specific primers for $N b E R F$ genes were designed and synthesized by GenScript (Nanjing) (product size $110-130 \mathrm{bp} ; \mathrm{Tm} 59^{\circ} \mathrm{C}-$ $61{ }^{\circ} \mathrm{C}, E F 1 \alpha$ was used as an internal control. All reactions were performed on an Icycleri Q5 system (Bio-Rad), using the SYBR Green SuperMix Kit (Vazyme Biotech) according to the manufacturer's instructions. The expression levels of these genes were calculated as $2^{-\Delta \mathrm{CT}}$ values. The relative temporal expression levels of each gene were also calculated as $2^{-\Delta \mathrm{CT}}$ values. At least three biological replicates were used for the fluorescencequantitative PCR reactions, with each biological repeat having at least three technical replicates.

\section{RNA sequence (RNA-seq) profiling}

The NbERF173-silenced N. benthamiana leaves at 6 hpi and the mock-treated leaves were selected for RNA-Seq analysis. Total RNA was isolated using the Trizol reagent (TIANGEN) according to the manufacturer's instructions and then treated with DNase I (RNase free, TaKaRa) to remove genomic DNA contaminations. The RNA samples from three biological replicates for each sample were pooled and sequenced with $100 \mathrm{bp}$ pairedend reads on Illumina HiSeq 2000 platform.

\section{Agrobacterium-mediated transient expression in $\mathbf{N}$. benthamiana leaves}

The cDNA fragments of NbERF173 and proteinase inhibitors (PIs) were cloned into pBINPLUS with a GFP-tag (Song et al. 2015). Transformation of Agrobacterium GV3101 by electroporation and infiltration of Agrobacterium suspensions were done as described by Asai et al. (2008).

\section{Subcellular localization of NbERF173}

To observe the subcellular localization of green fluorescent protein pBINPLUS:NbERF173 fusion protein, $A$. tumefaciens-mediated transient expression assays were used to express GFP (CK) and GFP:NbERF173 fusion proteins in $N$. benthamiana. After treatment for $48 \mathrm{~h}, N$. benthamiana leaves treated with $A$. tumefaciens were infiltrated with $5 \mathrm{mg} / \mathrm{mL}$ 4',6-diamidino-2-phenylindole (DAPI) solution. The infiltrated plant leaves were cut into small squares and mounted in water under a cover slip. Fluorescence and DAPI staining were visualized by epifluorescence microscopy using ultraviolet light. The excitation wavelength was $488 \mathrm{~nm}$ for GFP and $405 \mathrm{~nm}$ for DAPI (Song et al. 2015). The GFP transgenic $N$. benthamiana leaves were used as controls. 


\section{Virus-induced gene silencing}

VIGS of $N$. benthamiana was performed as described previously (Ratcliff et al. 2001). Briefly, an NbERF173 fragment exhibiting high sequence similarity to other members of this family was cloned in an antisense manner into the tobacco rattle virus (TRV) vector to create a silencing construct (pTRV:NbERF173). Agrobacterium strains harboring the TRV1 vector and TRV:GFP, TRV: PDS (phytoenedesaturase) or TRV:NbERF173 were mixed in a 1:1 ratio to achieve a final OD600 of 0.5 for each strain. The co-cultures were then infiltrated into the lower three leaves of 2-week-old plants. VIGS was done as described by Ratcliff et al. (2001). Additional file 10: Table S7 lists the primers used to amplify cDNA fragments from the $N$. benthamiana cDNA library (Yoshioka et al. 2003). Restriction sites were added to the $5^{\prime}$-ends of the forward and reverse primers for cloning into the TRV vector PTRV2.

\section{Phytophthora infection assays}

To assay the $P$. parasitica infection of gene-silenced $N$. benthamiana leaves 2 weeks after infiltrating Agrobacterium strains harboring vectors for VIGS, the upper leaves corresponding to the photo-bleached leaves in plants separately infected with TRV:PDS were detached and used for qRT-PCR analysis and $P$. parasitica inoculation assays. The lesion diameters were measured at 36 and 48 hpi. Significant differences were identified using $t$ tests. For the B. cinerea inoculation assays, the lesion diameters were measured at 36 and $48 \mathrm{hpi}$ and significant differences were identified using $t$-tests. To analyze pathogen development, the hyphal extension in the host cells was visualized by trypan blue staining (Yoshioka et al. 2003). At 24 hpi, abundant P. parasitica hyphae were observed in tissues of TRV:NbERF173 leaves.

\section{Supplementary information}

Supplementary information accompanies this paper at https://doi.org/10. 1186/s42483-020-0045-3.

Additional file 1: Table S1. The ERF family of $N$. benthamiana.

Additional file 2: Table S2. The RT-PCR results of some $N$. benthamiana ERF factors.

Additional file 3: Figure S1. Expression profiles of NbERF173 in leaves in response to salicylic acid (SA), jasmonic acid (JA) and ethylene (ETH) treatment. The expression levels at each time point were normalized by the expression levels in the samples treated with fresh water and harvested at the corresponding time point (control). Three biological replications were conducted. The error bars represent the standard deviation (*, $P<0.05 ;{ }^{* *} P<0.01$, Dunnett's test).

Additional file 4: Figure S2. Validation of gene silencing specificity. a Relative expressions of NbERF173 homologous. RNA-Seq data of NbERF236, NbERF244, NbERF246 and NbERF175 were compared in the NbERF173-silenced and control leaves. b VIGS-treated N. benthamiana seedlings. The $N$. benthamiana seedlings were treated with PTRV:PDS (a positive control to silence the phytoene desaturase gene line), PTRV:NbERF173 and pTRV:GFP (the negative control).
Additional file 5: Table S3. Eleven candidate genes were up-regulated by NbERF173.

Additional file 6: Table S4. Thirty-seven candidate genes were downregulated by NbERF173.

Additional file 7: Table S5. The downstream genes regulated by NbERF173.

Additional file 8: Figure S3. The relative expression levels of proteinase inhibitor I-B (P/1-B) and Kunitz trypsin inhibitor 1 (KT/1) were verified in the NbERF173-overexpressed and control leaves by qRT-PCR with EFla gene as a reference.

Additional file 9: Table S6. The promoter sequences of the two proteinase inhibitors.

Additional file 10: Figure S4. Yeast one-hybrid assay of NbERF173 binding to promoters of two PI genes. PPI1-B and PKTI indicate the promoters of the two Pl genes, respectively. The left yeast colonies showed that yeasts were grown on SD medium (-Leu-Trp). The right colonies indicated that yeasts were grown on SD medium (-His-Leu-Trp), suggesting of positive binding activities. The combination of pGAD53m/ pHIS2 was used as negative controls while the combination of pGAD53m/pHiS53 was used as positive controls.

Additional file 11: Table S7. The primers involved in this study.

Abbreviations

ERF: Ethylene response factor; KTI1: Kunitz trypsin inhibitor 1; PDS: Phytoene desaturase; PI1-B: Proteinase inhibitor I-B; TF: Transcription factor; TRV: Tobacco rattle virus; VIGS: Virus induced gene silencing; $\mathrm{Y} 1 \mathrm{H}$ : Yeast one-hybridization

\section{Acknowledgments}

Not applicable.

\section{Authors' contributions}

$J Y$ and CC conceived and designed the experiments. YJ and WL conducted the plant inoculation and RNA extraction. TB conducted the GRT-PCR validation and statistics analysis on experimental data. GA and XZ performed all the bioinformatics analysis. YC and DD provided technical support and edited the manuscript. All authors read and approved the final manuscript.

\section{Funding}

This work was supported by the National Natural Science Foundation of China (Grant No. 31625023) and Special Fund for Agro scientific Research in the Public Interest (Grant No. 201503112).

Availability of data and materials

The datasets used and/or analysed during the current study are available from the corresponding author on reasonable request.

Ethics approval and consent to participate

Not applicable.

Consent for publication

Not applicable.

\section{Competing interests}

The authors declare that they have no competing interests.

\section{Author details}

'Department of Plant Pathology, Nanjing Agricultural University, Nanjing 210095, China. ${ }^{2}$ Shandong Peanut Research Institute, Qingdao 266100, Shandong, China. ${ }^{3}$ College of Life Science and Technology, Nanyang Normal University, Nanyang 473061, China. 
Received: 19 August 2019 Accepted: 2 January 2020

\section{Published online: 31 January 2020}

\section{References}

Asai S, Ohta K, Yoshioka H. MAPK signaling regulates nitric oxide and NADPH oxidase-dependent oxidative bursts in Nicotiana benthamiana. Plant Cell. 2008:20:1390-406.

Bally J, Nakasugi K, Jia F, Jung H, Ho SYW, Wong M, et al. The extremophile Nicotiana benthamiana has traded viral defence for early vigour. Nat Plants. 2015;:1:15165

Benchabane M, Schlüter U, Vorster J, Goulet MC, Michaud D. Plant cystatins. Biochimie. 2010;92:1657-66.

Büttner M, Singh KB. Arabidopsis thaliana ethylene-responsive element binding protein (AtEBP), an ethylene-inducible, GCC box DNA-binding protein interacts with an ocs element binding protein. Proc Natl Acad Sci U S A. 1997:94:5961-6.

Carrillo L, Martinez M, Álvarez-Alfageme F, Castañera P, Smagghe G, Diaz I, et al. A barley cysteine-proteinase inhibitor reduces the performance of two aphid species in artificial diets and transgenic Arabidopsis plants. Transgenic Res. 2011;20:305-19.

Dong L, Cheng Y, Wu J, Cheng Q, Li W, Fan S, et al. Overexpression of GmERF5, a new member of the soybean EAR motif-containing ERF transcription factor, enhances resistance to Phytophthora sojae in soybean. J Exp Bot. 2015;66: 2635-47.

Dou D, Kale SD, Wang X, Jiang RHY, Bruce NA, Arredondo FD, et al. RXLRmediated entry of Phytophthora sojae effector Avr1b into soybean cells does not require pathogen-encoded machinery. Plant Cell. 2008;20:1930-47.

Feng J, Meyer CA, Wang Q, Liu JS, Liu SX, Zhang Y. GFOLD: a generalized fold change for ranking differentially expressed genes from RNA-seq data. Bioinformatics. 2012;28:2782-8.

Fujimoto SY, Ohta M, Usui A, Shinshi H, Ohme-Takagi M. Arabidopsis ethyleneresponsive element binding factors act as transcriptional activators or repressors of GCC box-mediated gene expression. Plant Cell. 2000;12:393404.

Goodin MM, Zaitlin D, Naidu RA, Lommel SA. Nicotiana benthamiana: its history and future as a model for plant-pathogen interactions. Mol Plant-Microbe Interac. 2008;21:1015-26.

Gutterson N, Reuber TL. Regulation of disease resistance pathways by AP2/ERF transcription factors. Curr Opin Plant Biol. 2004;7:465-71.

Hao D, Ohme-Takagi M, Sarai A. Unique mode of GCC box recognition by the DNA-binding domain of ethylene-responsive element-binding factor (ERF domain) in plant. J Biol Chem. 1998;273:26857-61.

Haq SK, Atif SM, Khan RH. Protein proteinase inhibitor genes in combat against insects, pests, and pathogens: natural and engineered phytoprotection. Arch Biochem Biophys. 2004;431:145-59.

Hu Y, Zhao L, Chong K, Wang T. Overexpression of OsERF1, a novel rice ERF gene, up-regulates ethylene-responsive genes expression besides affects growth and development in Arabidopsis. J Plant Physiol. 2008;165:1717-25.

Jin JH, Zhang HX, Tan JY, Yan MJ, Li DW, Khan A, et al. A new ethyleneresponsive factor CaPTII gene of pepper (Capsicum annuum L.) involved in the regulation of defense response to Phytophthora capsici. Front Plant Sci. 2015;6:1217.

Kamoun S, Furzer O, Jones JDG, Judelson HS, Ali GS, Dalio RJD, et al. The top 10 oomycete pathogens in molecular plant pathology. Mol Plant Pathol. 2015; 16:413-34.

Kim JY, Park SC, Hwang I, Cheong H, Nah JW, Hahm KS, et al. Protease inhibitors from plants with antimicrobial activity. Int J Mol Sci. 2009;10:2860-72.

Kroon LPNM, Brouwer H, de Cock AWAM, Govers F. The genus Phytophthora anno 2012. Phytopathology. 2012;102:348-64.

Lee JH, Hong JP, Oh SK, Lee S, Choi D, Kim W. The ethylene-responsive factor like protein 1 (CaERFLP1) of hot pepper (Capsicum annuum L.) interacts in vitro with both GCC and DRE/CRT sequences with different binding affinities: possible biological roles of CaERFLP1 in response to pathogen infection and high salinity conditions in transgenic tobacco plants. Plant Mol Biol. 2004;55: 61-81.

Li MY, Xu ZS, Huang Y, Tian C, Wang F, Xiong AS. Genome-wide analysis of AP2/ERF transcription factors in carrot (Daucus carota $\mathrm{L}$.) reveals evolution and expression profiles under abiotic stress. Mol Gen Genomics. 2015;290:2049-61.

Licausi F, Ohme-Takagi M, Perata P. APETALA2/ethylene responsive factor (AP2/ ERF) transcription factors: mediators of stress responses and developmental programs. New Phytol. 2013;199:639-49.
Liu J, Li J, Wang H, Fu Z, Liu J, Yu Y. Identification and expression analysis of ERF transcription factor genes in petunia during flower senescence and in response to hormone treatments. J Exp Bot. 2011;62:825-40.

Majeed A, Makhdoom R, Husnain T, Riazuddin S. Assessment of potato proteinase inhibitor-ll gene as an antifungal and insecticidal agent. Acta Agric Scand Sect B. 2011;61:92-6.

Meng Y, Zhang Q, Ding W, Shan W. Phytophthora parasitica: a model oomycete plant pathogen. Mycology. 2014;5(2):43-51.

Nakano T, Suzuki K, Fujimura T, Shinshi H. Genome-wide analysis of the ERF gene family in Arabidopsis and rice. Plant Physiol. 2006;140:411-32.

Okamuro JK, Caster B, Villarroel R, Montagu MV, Jofuku KD. The AP2 domain of APETALA2 defines a large new family of DNA binding proteins in Arabidopsis. Proc Natl Acad Sci U S A. 1997;94:7076-81.

Pirrello J, Jaimes-Miranda F, Sanchez-Ballesta MT, Tournier B, Khalil-Ahmad Q, Regad $F$, et al. SI-ERF2, a tomato ethylene response factor involved in ethylene response and seed germination. Plant Cell Physiol. 2006;47:1195-205.

Quilis J, Meynard D, Vila L, Avilés FX, Guiderdoni E, San SB. A potato carboxypeptidase inhibitor gene provides pathogen resistance in transgenic rice. Plant Biotechnol J. 2007:5:537-53.

Rashid M, He G, Yang G, Hussain J, Xu Y. AP2/ERF transcription factor in rice: genome-wide canvas and syntenic relationships between monocots and eudicots. Evol Bioinforma. 2012;8:321-55.

Ratcliff F, Martin-Hernandez AM, Baulcombe DC. Technical advance: tobacco rattle virus as a vector for analysis of gene function by silencing. Plant $\mathrm{J}$. 2001;25:237-45.

Ryan CA. Protease inhibitors in plants: genes for improving defenses against insects and pathogens. Annu Rev Phytopathol. 1990;28:425-49.

Sakuma Y, Liu Q, Dubouzet JG, Abe H, Shinozaki K, Yamaguchi-Shinozaki K. DNAbinding specificity of the ERF/AP2 domain of Arabidopsis DREBs, transcription factors involved in dehydration- and cold-inducible gene expression. Biochem Biophys Res Commun. 2002;290:998-1009.

Schluter U, Benchabane M, Munger A, Kiggundu A, Vorster J, Goulet MC, et al. Recombinant protease inhibitors for herbivore pest control: a multitrophic perspective. J Exp Bot. 2010;61:4169-83.

Senthilkumar R, Cheng CP, Yeh KW. Genetically pyramiding protease-inhibitor genes for dual broad-spectrum resistance against insect and phytopathogens in transgenic tobacco. Plant Biotechnol J. 2010;8:65-75.

Shen D, Chai C, Ma L, Zhang M, Dou D. Comparative RNA-Seq analysis of Nicotiana benthamiana in response to Phytophthora parasitica infection. Plant Growth Regul. 2016;80:59-67.

Shigyo M, Ito M. Analysis of gymnosperm two-AP2-domain-containing genes. Dev Genes Evol. 2004:214:105-14

Shin R, Park JM, An JM, Paek KH. Ectopic expression of Tsil in transgenic hot pepper plants enhances host resistance to viral, bacterial, and oomycete pathogens. Mol Plant-Microbe Interac. 2002;15:983-9.

Song T, Ma Z, Shen D, Li Q, Li W, Su L, et al. An oomycete CRN effector reprograms expression of plant HSP genes by targeting their promoters. PLoS Pathog. 2015;11:e1005348.

Swaminathan K, Peterson K, Jack T. The plant B3 superfamily. Trends Plant Sci. 2008;13:647-55.

Tamura K, Peterson D, Peterson N, Stecher G, Nei M, Kumar S. MEGA5: molecular evolutionary genetics analysis using maximum likelihood, evolutionary distance, and maximum parsimony methods. Mol Biol Evol. 2011;28:2731-9.

Thimm O, Bläsing O, Gibon Y, Nagel A, Meyer S, Krüger P, et al. MAPMAN: a userdriven tool to display genomics data sets onto diagrams of metabolic pathways and other biological processes. Plant J. 2004;37:914-39.

Tournier B, Sanchez-Ballesta MT, Jones B, Pesquet E, Regad F, Latché A, et al. New members of the tomato ERF family show specific expression pattern and diverse DNA-binding capacity to the GCC box element. FEBS Lett. 2003;550: $149-54$.

Trapnell C, Pachter L, Salzberg SL. TopHat: discovering splice junctions with RNASeq. Bioinformatics. 2009:25:1105-11.

Valdes-Rodriguez S, Cedro-Tanda A, Aguilar-Hernandez V, Cortes-Onofre E, Blanco-Labra A, Guerrero-Rangel A. Recombinant amaranth cystatin (AhCPI) inhibits the growth of phytopathogenic fungi. Plant Physiol Biochem. 2010; 48:469-75.

Valueva TA, Revina TA, Gvozdeva EL, Gerasimova NG, Ozeretskovskaia OL. Role of proteinase inhibitors in potato protection. Russ J Bioorg Chem. 2003;29:454-8.

Valueva TA, Revina TA, Kladnitskaya GV, Mosolov W. Kunitz-type proteinase inhibitors from intact and Phytophthora-infected potato tubers. FEBS Lett. 1998;426:131-4. 
Woloshuk CP, Meulenhoff JS, Sela-Buurlage M, van den Elzen PJ, Cornelissen BJ. Pathogen-induced proteins with inhibitory activity toward Phytophthora infestans. Plant Cell. 1991;3:619-28.

Yoshioka H, Numata N, Nakajima K, Katou S, Kawakita K, Rowland O, et al. Nicotiana benthamiana gp $91^{\text {phox }}$ homologs NbrbohA and NbrbohB participate in $\mathrm{H}_{2} \mathrm{O}_{2}$ accumulation and resistance to Phytophthora infestans. Plant Cell. 2003;15:706-18.

Zhang G, Chen M, Chen X, Xu Z, Guan S, Li LC, et al. Phylogeny, gene structures, and expression patterns of the ERF gene family in soybean (Glycine max L.). J Exp Bot. 2008;59:4095-107.

Zhang Z, Huang R. Enhanced tolerance to freezing in tobacco and tomato overexpressing transcription factor TERF2/LeERF2 is modulated by ethylene biosynthesis. Plant Mol Biol. 2010;73:241-9.

Zhao Y, Chang X, Qi D, Dong L, Wang G, Fan S, et al. A novel soybean ERF transcription factor, GmERF113, increases resistance to Phytophthora sojae infection in soybean. Front Plant Sci. 2017;8:299

Zhu X, Qi L, Liu X, Cai S, Xu H, Huang R, et al. The wheat ethylene response factor transcription factor pathogen-induced ERF1 mediates host responses to both the necrotrophic pathogen Rhizoctonia cerealis and freezing stresses. Plant Physiol. 2014;164:1499-514.

Ready to submit your research? Choose BMC and benefit from:

- fast, convenient online submission

- thorough peer review by experienced researchers in your field

- rapid publication on acceptance

- support for research data, including large and complex data types

- gold Open Access which fosters wider collaboration and increased citations

- maximum visibility for your research: over $100 \mathrm{M}$ website views per year

At $\mathrm{BMC}$, research is always in progress.

Learn more biomedcentral.com/submissions 